\title{
Normative Industrial Symbiotic Networks: A Position Paper
}

\author{
Vahid Yazdanpanah, Devrim Murat Yazan, and W. Henk M. Zijm \\ University of Twente, Enschede, The Netherlands \\ $\{$ v.yazdanpanah,d.m.yazan, w.h.m.zijm\}@utwente.nl
}

\begin{abstract}
In this paper, we introduce a normative, multi-agent perspective on the field of industrial symbiosis research and propose normative institutions as a key technology for operating Industrial Symbiotic Networks (ISNs), both as a framework to represent and reason about dynamic behaviour of ISNs and as a platform for design and maintenance of such networks. We discuss the requirements of normative agent-based frameworks for ISNs with respect to agent interactions, joint commitments, and the organisation to monitor interactions in ISNs.
\end{abstract}

\section{Introduction}

As a key concept in facilitated industrial practices, industrial symbiosis "engages traditionally separate industries in a collective approach to competitive advantage involving physical exchange of material, energy, water, and byproducts" [7]. Among various approaches that aim at providing a framework for representing and reasoning about industrial symbiosis, we encounter proposals with different perspectives. In [5], the interactions amongst industrial firms are seen as processes, the study of [17] has a statistical point of view which merely focuses on the case of the National Industrial Symbiosis Programme (NISP) in the UK [14], and [23] is focused on organizational perspectives. One point of agreement among these studies is the dynamic nature of industrial symbiosis. In other words, an Industrial Symbiotic Network (ISN) is not a fixed and static institution but a dynamic and evolving one. In this respect, one significant contribution that clearly goes beyond the traditional definition of industrial symbiosis by Chertow [7], is the study of [16]. In the latter, the main attempt is to provide a more relaxed and dynamic definition for industrial symbiosis which is not limited to geographical proximity and is broader than the focus on waste-resource exchanges only. We see that this definition is successful in describing the behaviour of ISNs that are based on sharing both tangible and intangible assets. However, more work needs to be done in tailoring it for specifying the dynamics of the complex behaviour of ISNs, regarding temporal as- 
pects. For instance, an ISN that is operating now might face different economic circumstances (e.g., market price) as well as structural settings (e.g., entrance of new ISN members) in a later stage. These possible changes can influence the efficiency and stability of ISNs over time. Roughly speaking, is an ISN today still an ISN tomorrow or the next quarter? We claim that answering such a question, necessitates modelling approaches that incorporate the long-term behaviour and subtleties of temporal behaviour of ISNs.

Presence of multiple decision makers and heterogeneity of industrial firms with respect to their interests and preferences make multi-agent systems a natural modelling paradigm for formal specification and verification of the properties of such networks. Moreover, ISNs are not aiming to merge industrial firms but to establish a loose bounding and control. Hence, we believe that applying formal modelling approaches in the mature field of normative multi-agent systems and norm-based coordination mechanisms can result in frameworks that are expressive enough to represent and reason about multi-dimensional behaviour of ISNs. To our knowledge, although some studies on industrial symbiosis, e.g. [3, 1], consider the agent-based paradigm, they merely focus on agent-based simulation and scenario analysis. As part of the European Union's Horizon 2020 research and innovation programme, the SHARE-
$B O X$ project focuses, among other things, on the analysis, modelling, design and maintenance of ISNs and on decision-support tools that enable secure operation of such networks. In this position statement, we (1) see ISNs as instances of normative multi-agent systems [4], (2) discuss the requirements that need to be taken into account to formalize normative ISNs, and (3) introduce norm-aware institutions as a coordination mechanism for ISNs. Normative agentbased approaches has been successfully used for specification and verification of multi-agent organizations $[21,6,8]$. We now build on such well-established frameworks and propose a similar approach to specify, analyse and manage ISNs, both as platforms for designing new instances and as logical platforms to analyse and reason about the behaviour of existing ISNs.

The remainder of this paper is organized as follows. In Section 2, we provide a general analysis of our normative conception of industrial symbiosis. In Sections 3 and 4 , we discuss agent interactions in ISNs, explain modelling requirements to express joint agreements, and introduce regulatory institutions to coordinate ISNs. Finally, we note some challenging issues in modelling the complex behaviour of ISNs in Section 5 and conclude in Section 6. 


\section{Normative Industrial Symbiosis: Conceptual Analysis}

Imagine a realistic scenario ${ }^{1}$ in which five industrial firms, represented by agents $^{2} i, j, k, l$, and $m$, are located and active in an industrial region where $i$ and $j$ are metal industries, $k$ is a recycling plant, and both $l$ and $m$ are chemical industries (Figure 1). The two metal industries have zinc waste as their main waste that was traditionally disposed at high cost. Moreover, the two chemical industries $l$ and $m$ have regular demand for zinc powder as their main primary input. On the other hand, both $l$ and $m$ have excess steam waste that is traditionally disposed to the environment while $i$ and $j$ are using turbines that require high amounts of steam. In this case, a reasonable solution that is both economically and environmentally beneficial for all the involved industries is to establish an industrial symbiotic relation that involves: a long-term collaboration for exchanging resources, an agreement in which the rules of collaboration are stated, and a secure mechanism that guarantees the maintenance and security of such a collaboration.

Communication is essential and distinguishable: Let us assume that the involved agents in this symbiosis scenario have become conscious of the benefits of a long- term collaboration and are willing to make commitments to each other. For instance, $m$ offers that it can deliver a specific amount of steam per month to $j$ if $j$ delivers a specific amount of zinc waste per month to $k$. We highlight that such an offer does not affect the industrial environment. In other words, the offer of $m$ to deliver an amount is distinguishable from the act of delivering. However, it is not rational that $m$ just delivers such an amount before first offering it and getting the confirmation that $j$ accepts the offer. We say that for long-term collaboration (and not a spontaneous industrial interaction) the communication actions between the involved agents are necessary. As framed by Grosz [13], "collaboration requires communication". Thus, we make distinction between industrial actions that affect the state of environment and communication actions. Such a distinction is in-line with Searle's fundamental approach to define institutions and his distinction between physical and institutional actions [19].

Collaboration agreements: In order to enable the regulation of the collaborative relations among the involved industries in a multiagent ISN, a formalism to represent the mutual agreements is required. We argue that due to the dynamicity of the industrial context and the possible strategic behaviour of industrial agents,

\footnotetext{
${ }^{1}$ This scenario is adapted from an ISN located at Ulsan, South Korea [18].

${ }^{2}$ In this paper, we simplify industrial firms and represent any industry as a single industrial agent. I.e., we dismiss the decision-making processes within each firm and focus on the interactions amongst industries.
} 


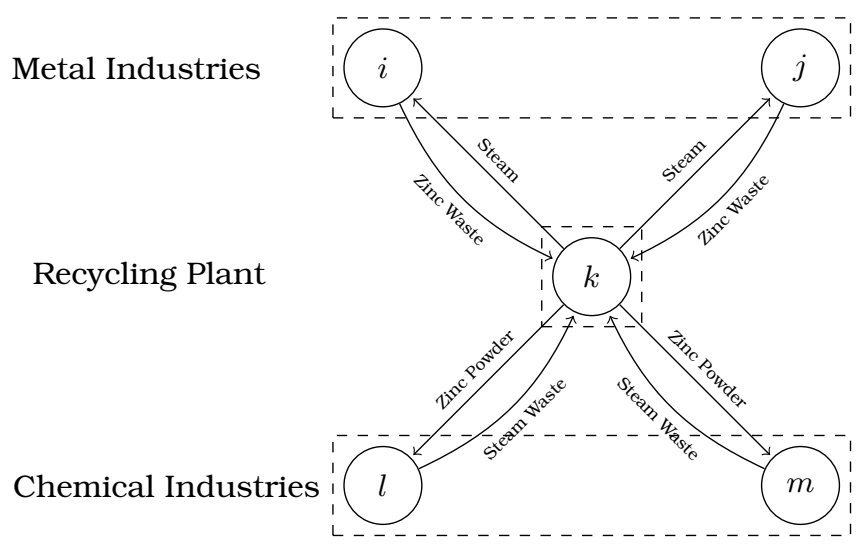

Fig. 1. Industrial Symbiosis Scenario

static contracts and regimentbased approaches that impose pre-determined restrictions on the actions of industries may not be effective. We follow [22,9] in their argument that, in order to stabilize the collaboration (as a desired situation), it is not efficient to impose constraints on unwanted behaviour. But an apt solution would be: to define norms that reflect the goals of the industrial symbiosis, to detect violations, and to react to violations. For example, if $j$ accepts the above-mentioned offer from $m$, agent $m$ is expected to follow the norm (to collaborate) and to deliver the promised resource as stated in its offer. More general, collaborative agreement of an industrial symbiosis can be expressed as a set of formal propositions that denote the joint commitments of industrial agents.

Normative institution: In the industrial context in which a set of heterogeneous agents may become involved in an ISN, agent's deviations from the desired behaviours may occur due to various reasons, e.g., strategic behaviour of involved industries. Hence, in order to maintain industrial symbiosis and to incentivize external industries to join such a platform, mechanisms to systematize and secure the set of desired behaviours are essential. For such a purpose a normative institution is widely proposed as a solution concept for coordination and regulation of agent behaviour [2, 4, 22]. In such an institution, a set of norms (that could be specified as normative rules) aims to enforce the goals of the institution. On the other hand, individual agents update the state of the environment (mainly) by means of performing industrial acts or update the state of the institution (mainly) by means of performing institutional acts. For example, after the above-mentioned offer (from $m$ ), agent $j$ may confirm that it accepts the offer. Such a confirmation by $j$ in combination with the former offer by $m$ can be counted as the 
establishment of a joint commitment between $m$ and $j$. Such a set of rules, e.g., the rule that the combination of a well-defined offer and a related confirmation counts as the establishment of a joint commitment, forms the first regulating component of our proposed representation of industrial symbiosis as a normative institution. Now, imagine that $m$ (despite the joint commitment) violates the norm and refuses to deliver the resources. In such cases (of norm violation) the institution can react by applying sanction rules [6]. E.g., refusing to bring about the situations that are mentioned in a joint commitment can result in being disqualified for industrial trade for a given amount of time. The set of sanction rules forms the second regulatory component of our normative industrial symbiosis framework.

\section{Industry Interactions and Joint Commitments}

Possible actions that industrial agents can perform in an ISN to influence their environment or to interact with each other consist of two exclusive sets of actions: communication actions, e.g., offering another firm that a specific amount of resources can be delivered by a specific deadline, and industrial actions, e.g., delivering a specific amount of resources to another firm subject to specified conditions. Roughly speaking, industrial actions influence the actual state of the shared industrial envi- ronment amongst industrial firms (involved in an ISN) while communication actions do not influence the state of the industrial environment. For modeling the agent interactions in an ISN, we assume a set of involved agents in the ISN, a set of propositional variables that reflect the possible states in the industrial environment, and a domain-specific set of legal communication phrases. Accordingly, a communication action can be seen as the apprising of a legal communication phrase by one of the agents in the ISN towards another member of ISN while an industrial action by an agent (in the ISN) would be to bring about a propositional variable. E.g., in our ISN scenario, the communication act of agent $m$, offering the monthly delivery (mon) of steam waste $(s w)$ to the recycling plant $k$, can be represented by the communication phrase of$\operatorname{fer}(m, k, m o n, s w)$. Our approach to limit communication phrases to a set of sufficiently expressive phrases follows the approach of [8]. Moreover, our action categorization correlate with classification of institutional and physical acts in the sense of [19].

One aspect that we consider crucial as a distinction between ISN practices and traditional business-to-business relations is the duration that such practices last. In our view, an industryindustry relation should not be called industrial symbiosis if it basically occurs in reaction to a spontaneous industrial need (for a primary input) but has no concern 
for a long-term relationship between the involved industries. In order to specify and maintain the long-term relationships amongst agents, commitment-based approaches [11] propose a deontic perspective in which agents commit to other agents that they bring about a specific proposition before a given due or/and with respect to the occurrence of some other conditions. The fundamental work of Telang and Singh [21] tailors this commitment-based approach for cross-organizational business models. In a general form, they say that a debtor agent commits to a creditor agent that if the creditor brings about a given proposition $p$ by a specific due, the debtor will bring about a given proposition $q$ by a specific due. We follow their method in modelling joint commitments in ISNs and assume a set of involved agents in the ISN, a set of propositional variables that reflect the possible states in the industrial environment, and a set of integer deadlines. Accordingly, a given joint commitment between two agents in an ISN specifies that the debtor agent is committed to bring about a given propositional variable $p$ before deadline $d_{1}$ if the creditor agent brings about a given propositional variable $q$ before deadline $d_{2}$.

\section{Industrial Symbiosis Institution}

Following our proposal to model ISNs using commitment-based agreement technologies, we see the necessity to securely manage and maintain the well-being and (as defined by [7]) the "[. . .] competitive advantages involving physical exchange of materials, energy, water and by-products [...]" in ISNs. Hence, we need mechanisms to coordinate the behaviour of involved agents in ISNs and to enforce desired behaviours, e.g., the compliance of agents to joint commitments. There exists several multiagent frameworks (see [20, 10, 12]) proposing normative institutions as a solution concept for enforcing desired behaviours in electronic/trading institutions. We follow this line of research and propose a normative institution, specified by facts, norms, and sanctions, as a concept that enables the self-organization of ISNs.

In brief, the fact component, which consists of institutional and industrial facts, reflects the state of the institution, e.g., the set of already established commitments. In the norm component, the set of normative rules that relate industrial/institutional acts to (updated) industrial/institutional facts will be specified. This component reflects the desired behaviours and in a sense the way that an ISN designer expects that her ISN will work. Finally, sanction rules in the third component specify the sanctions to be introduced in case of norm violation by the involved agents in an ISN. In our ISN scenario, one norm instance could be that if an agent $i$ accepts an offer of $j$, agent $j$ should bring about the offer, e.g., should deliver the offered resource before a specific deadline. In this case, 
acting otherwise will be considered as a norm-violating behaviour (by $j$ ) and triggers the sanction rule (against $j$ ). The fact component can be programmed using propositional variables while both the norm and sanction rules can be expressed as Searle's count-as rules [19]. Our goal is to provide a full description of such ISNtailored normative notions (formal specification, dynamics and desired properties) in future work.

\section{Discussion: Modelling the Complex Behaviour of ISNs}

As noted earlier, the design and management of ISNs must consider various issues such as the behaviour over time of this industrial multi-agent system (temporal aspects) as well as mechanisms to monitor (and ensure) the commitment of involved industries to organizational objectives of the industrial symbiosis (coordination and control mechanisms). Fulfilling such necessities asks for comprehensive modelling frameworks, reasoning languages, and operational semantics to represent ISNs and to analyse their behaviour. In the following, we discuss some of the dimensions that we believe a formal model of ISNs should take into account. We view industrial firms that are involved in an ISN as agents with a high level of autonomy regarding their decisionmaking. In such a system, regulations provided by industrial symbiosis cannot intervene but can exogenously monitor the behaviour of agents and can only impose coordination policies, e.g., sanctions, in case of observing a violation. Accordingly, we see formal normative concepts (e.g., compliance and violation), suitable notions to formulate needed operational semantics for ISNs. Moreover, normative platforms such as proposed in [21] and [8] provide notions that need to be tailored: (1) for reasoning about the temporal properties of ISNs and (2) to formulate a logical characterization of the discussed concepts in this paper.

Due to the involvement of multiple agents in ISNs and their possible conflict of preferences, analysing the coalitional capacities of the possible sub-groups would be helpful for implementing a collusion-proof mechanism to supervise and maintain ISNs. For example, in our industrial symbiosis scenario, imagine a case in which the two zinc waste providers attempt to refuse to deliver for a specific period. Such (undesired) group decisions can strongly influence the efficiency of ISNs. Then the challenge for the industrial symbiosis designer will be to evaluate the effectiveness of the sanction rules to avoid such an undesired possibility. Therefore, sanction rules must be designed with respect to coalitional capacities of the involved agents. Otherwise, the ISN will be vulnerable to collusional actions. Moreover, considering coalitional capacities and strategy proofness of an ISN incentivizes newcomers to join and benefit from the collaboration in a secure fashion. 
A final key aspect concerns the relation of a given ISN member with industries that are not involved in the ISN. It is essential to design communication protocols to efficiently communicate and interact with agents that are not (yet) a member of an ISN. This approach relates our work to the line of research on the concept of Industrial Ecology (IE) [15]. In this sense, we see any ISN as a loosely coupled subset of IE that agrees to collaborate (internally) based on a specific agreement technology; however, it is able to relate to exogenous industrial agents that are active in the IE as its (external) industrial environment.

\section{Conclusion}

We have proposed a normative perspective and discussed requirements to be taken into account for formal representation and design of ISNs as institutions. Our proposal distinguishes between industrial and communication actions of the involved agents, follows a dynamic formalism by which the joint commitments of industrial agents can be expressed, and uses norm and sanction rules to regulate agent interactions and to avoid commitment violations. Although we discussed ISNs in the institutional level, the decisionmaking process of each industrial agent remains unresolved. This is how an industrial agent decides to offer to another agent or accept one. One alternative to deal with such decisions should be a rank of industries, with respect to the preferences of the ranker, which allows agents to choose the best ones to make an agreement with. For future work, we aim to apply methods from normative multi-agent systems to industrial symbiosis research and focus on formalizing operational semantics and designing a platform for analyzing the temporal behaviour of ISNs in strategic settings.

Acknowledgement: This research is funded by European Union's Horizon 2020 programme under grant agreement No. 680843.

\section{References}

1. Albino, V., Fraccascia, L., Giannoccaro, I.: Exploring the role of contracts to support the emergence of self-organized industrial symbiosis networks: an agent-based simulation study. Journal of Cleaner Production 112, 43534366 (2016)

2. Andrighetto, G., Governatori, G., Noriega, P., van der Torre, L.W.: Normative multi-agent systems, vol. 4. Schloss Dagstuhl-Leibniz-Zentrum fuer Informatik (2013)

3. Batten, D.F.: Fostering industrial symbiosis with agent-based simulation and participatory modeling. Journal of Industrial Ecology 13(2), 197-213 (2009)

4. Boella, G., Van Der Torre, L., Verhagen, H.: Introduction to normative multiagent systems. Computational \& Mathematical Organization Theory 12(23), 71-79 (2006) 
5. Boons, F., Spekkink, W., Jiao, W.: A process perspective on industrial symbiosis. Journal of Industrial Ecology 18(3), 341-355 (2014)

6. Bulling, N., Dastani, M.: Verifying normative behaviour via normative mechanism design. In: IJCAI. vol. 11, pp. 103-108 (2011)

7. Chertow, M.R.: Industrial symbiosis: literature and taxonomy. Annual review of energy and the environment 25(1), 313-337 (2000)

8. Dastani, M., van der Torre, L., Yorke-Smith, N.: Commitments and interaction norms in organisations. Autonomous Agents and Multi-Agent Systems pp. 1-43 (2015)

9. Dignum, V., Dignum, F.: Modelling agent societies: Co-ordination frameworks and institutions. In: Portuguese Conference on Artificial Intelligence. pp. 191-204. Springer (2001)

10. Dignum, V., Vázquez-Salceda, J., Dignum, F.: Omni: Introducing social structure, norms and ontologies into agent organizations. In: International Workshop on Programming Multi-Agent Systems. pp. 181-198. Springer (2004)

11. Fornara, N., Colombetti, M.: A commitment-based approach to agent communication. Applied Artificial Intelligence 18(9-10), 853-866 (2004)

12. García-Camino, A., Noriega, P., Rodríguez-Aguilar, J.A.: Implementing norms in electronic institutions. In: Proceedings of the fourth international joint conference on Autonomous agents and multiagent systems. pp. 667673. ACM (2005)

13. Grosz, B.J.: The contexts of collaboration. In: Cognition, Agency and Rationality, pp. 175-187. Springer (1999)

14. Laybourn, P., Clark, W.: National industrial symbiosis programme: a year of achievement. NISP (National Industrial Symbiosis Programme) (2004)

15. Lifset, R., Graedel, T.E.: Industrial ecology: goals and definitions. A handbook of industrial ecology pp. 3-15 (2002)

16. Lombardi, D.R., Laybourn, P.: Redefining industrial symbiosis. Journal of Industrial Ecology 16(1), 28-37 (2012)

17. Paquin, R.L., Howard-Grenville, J.: The evolution of facilitated industrial symbiosis. Journal of industrial Ecology 16(1), 83-93 (2012)

18. Park, H.S., Behera, S.K.: Methodological aspects of applying eco-efficiency indicators to industrial symbiosis networks. Journal of Cleaner Production 64, 478-485 (2014)

19. Searle, J.R.: The construction of social reality. Simon and Schuster (1995)

20. Sierra, C., Rodriguez-Aguilar, J.A., Noriega, P., Esteva, M., Arcos, J.L.: Engineering multi-agent systems as electronic institutions. European Journal for the Informatics Professional 4(4), 33-39 (2004)

21. Telang, P.R., Singh, M.P.: Specifying and verifying cross-organizational business models: An agent-oriented approach. IEEE Transactions on Services Computing 5(3), 305-318 (2012)

22. Vázquez-Salceda, J., Aldewereld, H., Dignum, F.: Implementing norms in multiagent systems. In: German Conference on Multiagent System Technologies. pp. 313-327. Springer (2004)

23. Walls, J.L., Paquin, R.L.: Organizational perspectives of industrial symbiosis: A review and synthesis. Organization \& Environment 28(1), 32-53 (2015) 